\title{
Comparison of quality of automated gene network reconstruction using connectivity of random and functional networks
}

\author{
E. Tiys ${ }^{1,2 *}$, P. Demenkov ${ }^{1}$, V. Ivanisenko ${ }^{1}$ \\ ${ }^{1}$ Institute of Cytology and Genetics SB RAS, Novosibirsk, Russia \\ ${ }^{2}$ Novosibirsk State University, Novosibirsk, Russia \\ *e-mail: tiys@bionet.nsc.ru
}

Key words: associative gene networks, network reconstruction, random networks, modularity

Motivation and Aim: Hartwell in 1999 drew attention to the modularity of biological systems [1]. Later it was shown that at the level of gene networks, modularity manifests itself in more connections between genes taken from one biological process, than from different [2]. Often biological experiment gives a set of genes with the differential characteristics and the task of the researcher is to determine the mechanisms, underlying the flow of the biological process. Such a gene set can be a starting set for reconstruction of the gene network for this process (associative gene network). Knowledge of the modular organization of biological systems can be used to assess the quality of the automated network reconstruction method.

Methods and Algorithms: In order to compare methods of gene network reconstruction, we used ROC-curves taken from [2], based on a positive sample of genes sets of size 10 of GeneOntology biological processes and a negative sample including sets of randomly selected genes. As a measure of the quality of network reconstruction, Area under ROCCurve (AUC) was chosen. As a reconstruction tool, we used ANDSystem. We used two simplest methods of reconstruction, level 0 network, including links only between the starting genes obtained in the experiment and level 1 network, which includes, in addition to the level 0 network, all the vertices adjacent to the level 0 network and all the links between them. P-value of the difference in the AUC of the ROC-curves was estimated using the two-sided unpaired DeLong's test, through the roc.test function of R language. Results: The method of reconstruction of level 0 network showed a significantly higher AUC value than level 1 network $(0.88$ vs. $0.61, \mathrm{p}<1.00 \mathrm{e}-05)$ and is estimated as a more promising automated method for reconstruction of associative gene networks.

Conclusion: This work is the first attempt to introduce an objective approach to comparing methods of automated reconstruction of associative gene networks.

Acknowledgements: Supported by the budget project No. 0324-2018-0017 and by integrational project No. 0324-2018-0021.

\section{References}

1. Hartwell L.H. et al. (1999) From molecular to modular cell biology. Nature. 402(Suppl 6761):C47-C52.

2. Tiys E.S., Ivanisenko T.V., Demenkov P.S., Ivanisenko V.A. (2018) FunGeneNet: a web tool to estimate enrichment of functional interactions in experimental gene sets. BMC Genomics. 19(Suppl 3):76. 